\title{
A one pot synthesis of fused chromenones
}

\author{
Kaushal P. Patel \\ Department of Advanced Organic Chemistry, P.D.Patel Institute of Applied Sciences, Charotar \\ University of Science and Technology, Charusat, Changa-388 421, Gujarat, India \\ E-mail: kaus_chem@yahoo.com
}

\begin{abstract}
A new class of compounds, 7-hydroxy-9,10-dihydroindeno[5,4-c]chromene-6,11-dione 2a-e, 7hydroxy-10,11-dihydro-6H-naphtho[2,1-c]chromene-6,12(9H)-dione 2f-j and 7-hydroxy-10,10dimethyl-10,11-dihydro-6H-naphtho[2,1-c]chromene-6,12(9H)-dione 2k-o have been synthesized by reacting various 1-(2-oxo-2-(2-oxo- $2 H$-chromen-3-yl)ethyl)pyridinium bromides 1a-e with 1,3-cyclopentandione, 1,3-cyclohexandione and dimedone respectively in the presence of sodium acetate in refluxing glacial acetic acid. Thus, new fused chromenones derivatives are synthesized and characterized by analytical and spectral data.
\end{abstract}

Keywords: 1-(2-Oxo-2-(2-oxo-2H-chromen-3-yl)ethyl)pyridinium bromide , indeno[5,4$c]$ chromene, naphtho[2,1-c]chromene

\section{Introduction}

During the last twenty years, the study of the biological activities of chromene derivatives has drawn the attention of many scientists. ${ }^{1-10}$ Recently, the anticoagulant, antibacterial, antihelminthic, hypothermal and vasodilatory properties of chromene have been reviewed. ${ }^{1}$ Fused chromenones are interesting due to their significant antibacterial ${ }^{11-15}$ and novobiocin ${ }^{16,17}$ activities. Recently, Selectfluor ${ }^{18}$ has been used as an alternative to conventional catalysts for the synthesis of substituted chromenones via Pechmann condensation of phenols with $\beta$-ketoesters under solvent-free conditions. Some of the co-workers developed simple and efficient synthesis of polyfunction heterocyclics from readily available starting materials. ${ }^{19,20}$ They have reported the synthesis of chromenopyridine and thiopyranochromene derivatives by cycloaddition of active methylene compounds with chromene-3-(4-aminosulfonyl) carbanilide ${ }^{19}$ or coumarin-3thiocarboxamide. ${ }^{20}$ Thus, considering the above synthetic methodology to prepare chromenones and its biological importance it was thought worthwhile to incorporate chromenone nucleus as a fuse group with indanone and naphthelenone. Therefore, in the present work we report a one pot synthesis of 7-hydroxy-9,10-dihydroindeno[5,4-c]chromene-6,11-dione 2a-e, 7-hydroxy-10,11- 
dihydro-6H-naphtho[2,1-c]chromene-6,12(9H)-dione 2f-j and 7-hydroxy-10,10-dimethyl-10,11dihydro-6H-naphtho[2,1-c]chromene-6,12(9H)-dione 2k-o utilizing inexpensive and easily available starting materials.

\section{Result and Discussion}

In this work, 1-(2-oxo-2-(2-oxo-2H-chromen-3-yl)ethyl)pyridinium bromide 1a-e were reacted with appropriate diketone compound such as 1,3-cyclopentandione, 1,3-cyclohexandione and dimedone respectively in the presence of sodium acetate in refluxing acetic acid to afford 7-hydroxy-9,10-dihydroindeno[5,4-c]chromene-6,11-dione 2a-e, 7-hydroxy-10,11-dihydro-6Hnaphtho[2,1-c]chromene-6,12(9H)-dione 2f-j and 7-hydroxy-10,10-dimethyl-10,11-dihydro-6Hnaphtho[2,1-c]chromene-6,12(9H)-dione 2k-o respectively (Scheme 1).

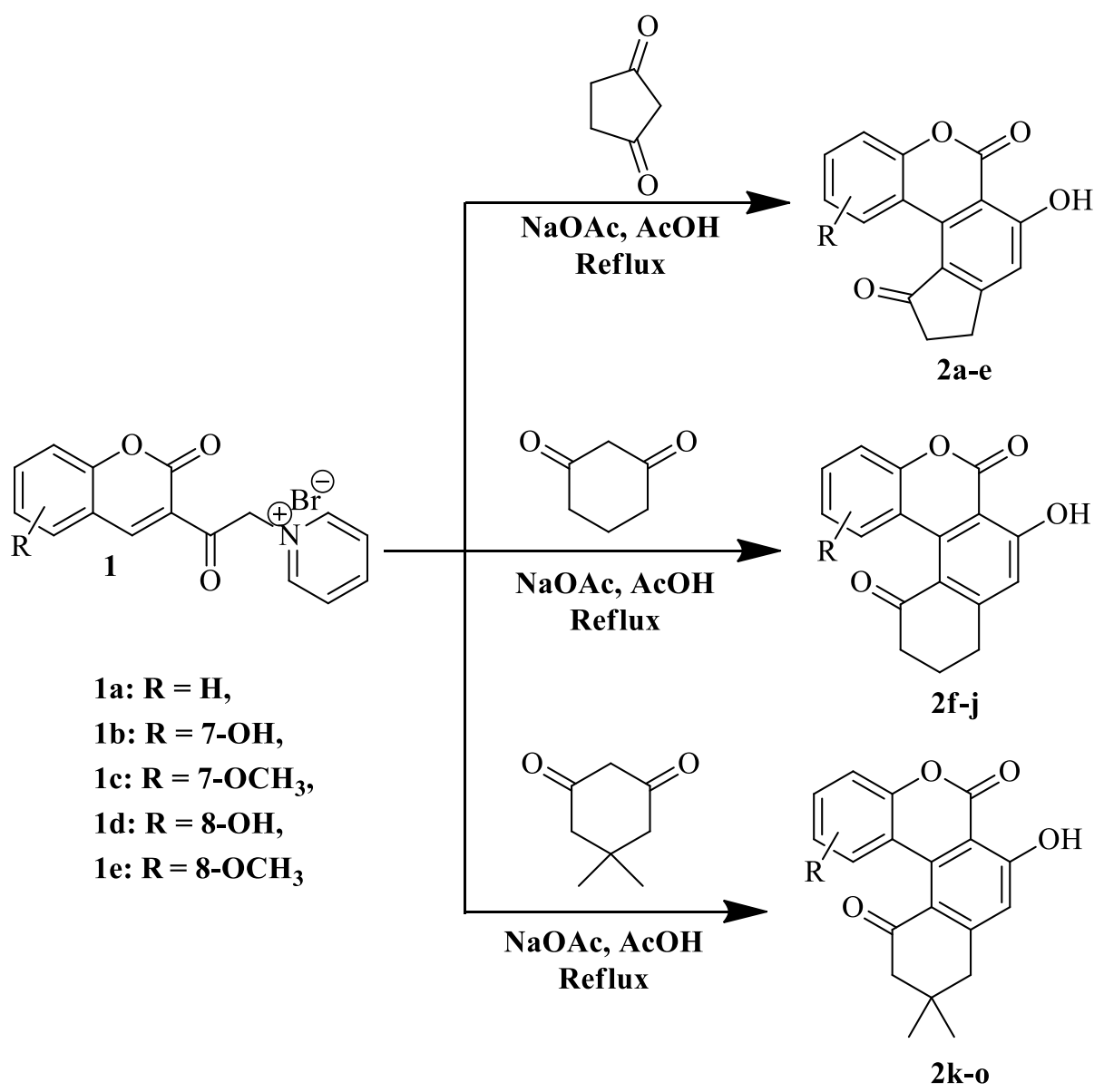

\section{Scheme 1}

Compound 1 was allowed to react with the 1,3-diketone under acidic conditions, to obtain fused chromenones $\mathbf{2}$. The reaction pathway is assumed to proceed by Michael addition of the 
active methylene function of 1-(2-oxo-2-(2-oxo- $2 \mathrm{H}$-chromen-3-yl) ethyl) pyridinium bromide on 1,3-diketone, resulting in the formation of intermediate having 1,5-dione functionality. The active methylene group (flanked by carbonyl ketone and pyridine moiety) then gets cyclized with carbonyl group of 1,3-diketone and the resultant intermediate finally aromatized to afford the product 2. The proposed mechanism is shown in Scheme 2.<smiles>O=C(O)CC1C(=O)CCCC1=O</smiles>

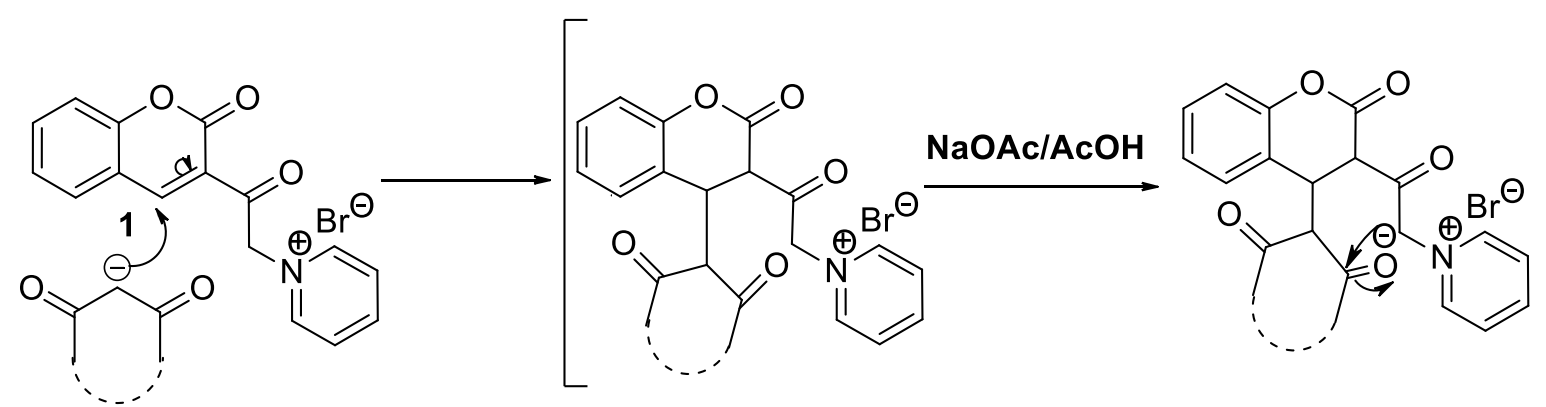

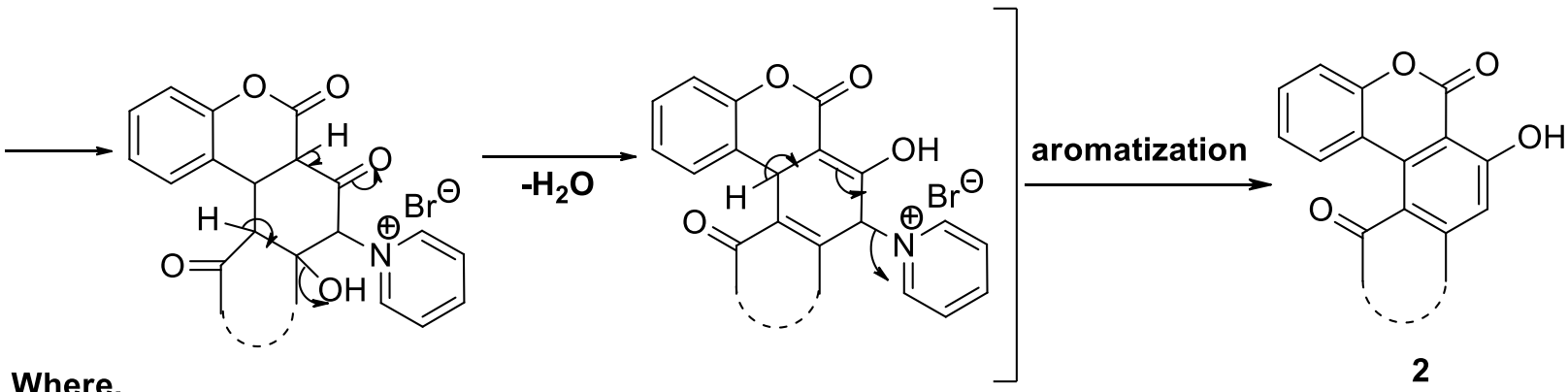

Where,<smiles>CC1(C)CC(=O)CC(=O)C1</smiles>

\section{Scheme 2}

The structures of all the synthesized compounds were established on the basis of IR, ${ }^{1} \mathrm{H}-$ NMR, ${ }^{13} \mathrm{C}-\mathrm{NMR}$, DEPT-135 spectral data, elemental analysis and molecular weights of some selected compounds $\mathbf{2 a}, \mathbf{2} \mathbf{f}$ and $\mathbf{2} \mathbf{k}$ were confirmed by mass spectrometry.

The IR spectrum of 2a-o showed characteristic bands around 1670, 1715, 2926, 1610, and $3020 \mathrm{~cm}^{-1}$ for carbonyl stretching vibrations of $\delta$-lactone ring, carbonyl stretching vibrations of 1,3-diketone ring, aliphatic $\mathrm{C}-\mathrm{H}$ stretching vibrations of $\mathrm{CH}_{2}$ groups, aromatic $\mathrm{C}=\mathrm{C}$ and $\mathrm{C}-\mathrm{H}$ stretching vibrations respectively. The decrease in $\mathrm{C}=\mathrm{O}$ stretching frequency of $\delta$-lactone ring from the normal value $\left(\sim 1710 \mathrm{~cm}^{-1}\right)$ is due to hydrogen bonding with $\mathrm{C}_{7}-\mathrm{OH}$. A broad band observed around $3440 \mathrm{~cm}^{-1}$ is due to phenolic-OH stretching. 
The NMR spectrum of compounds $\mathbf{2 a - e}$ showed two triplets around $\delta 3.08$ and $\delta 3.13$ each integrating for two protons attached at $\mathrm{C}_{9}$ and $\mathrm{C}_{10}$ respectively. A singlet appeared around $\delta 8.10$ is due to $\mathrm{C}_{8}-\mathrm{H}$ and $-\mathrm{OH}$ proton was seen as a broad singlet around $\delta 11.35$, which was conformed by $\mathrm{D}_{2} \mathrm{O}$ exchanged spectrum. The remaining aromatic protons appeared at appropriate positions and with appropriate multiplicity. The ${ }^{13} \mathrm{C}-\mathrm{NMR}$ spectra of compounds $\mathbf{2 a}-$ e showed signals around $\delta 28.0$ and 29.0 due to $C_{9}$ and $C_{10}$, respectively. This was further confirmed by DEPT-135 spectra in which these signals got inverted. This supports the incorporation of indanone ring in the compounds $\mathbf{2 a - e}$. The carbonyl carbon signals in indanone ring and $\delta$-lactone ring appeared around $\delta 200.0$ and $\delta 163.0$ respectively. The aromatic carbons appeared between $\delta 105.0$ and 161.6. The signal around $\delta 161.6$ is due to $\mathrm{C}_{7-\mathrm{OH}} \mathrm{O}$. Mass spectra of compound $\mathbf{2 a}$ gave molecular ion peak at $266.0\left(\mathrm{M}^{+}\right)$corresponding to molecular formula $\mathrm{C}_{16} \mathrm{H}_{10} \mathrm{O}_{4}$.

The NMR spectrum of compounds $\mathbf{2 f}-\mathbf{j}$ showed a multiplet around $\delta 2.25$ integrating for two protons attached at $\mathrm{C}_{10}$. Two triplets appeared around $\delta 2.70$ and $\delta 2.95$ each integrating for two protons attached at $\mathrm{C}_{9}$ and $\mathrm{C}_{11}$ respectively. A singlet appeared around $\delta 8.15$ is due to $\mathrm{C}_{8}-\mathrm{H}$ and $-\mathrm{OH}$ proton was seen as a broad singlet around $\delta 11.40$, which was conformed by $\mathrm{D}_{2} \mathrm{O}$ exchanged spectrum. The remaining aromatic protons appeared as expected. The ${ }^{13} \mathrm{C}-\mathrm{NMR}$ spectra of compounds $\mathbf{2 f}-\mathbf{j}$ showed signals around $\delta 23.0$, 30.0 and 35.0 due to $\mathrm{C}_{9}, \mathrm{C}_{10}$ and $\mathrm{C}_{11}$ respectively. This was further confirmed by DEPT-135 spectra in which these signals got inverted. This supports the incorporation of naphthalenone ring in the compounds $\mathbf{2} \mathbf{f}-\mathbf{j}$. The carbonyl carbon signals in naphthalenone ring and $\delta$-lactone ring appeared around $\delta 197.0$ and $\delta$ 163.0 respectively. The aromatic carbons appeared between $\delta 107.0$ and 161.9. The signal around $\delta 161.9$ is due to $\mathrm{C}_{7}-\mathrm{OH}$. Mass spectra of compound $\mathbf{2 f}$ gave molecular ion peak at 280.0 $\left(\mathrm{M}^{+}\right)$corresponding to molecular formula $\mathrm{C}_{17} \mathrm{H}_{12} \mathrm{O}_{4}$.

The NMR spectrum of compounds $\mathbf{2 k - 0}$ showed three singlets around $\delta 1.11,2.70$ and 2.90 due to six protons of two methyl groups attached at $\mathrm{C}_{10}$, two protons attached at $\mathrm{C}_{9}$ and $\mathrm{C}_{11}$ respectively. A singlet appeared around $\delta 8.20$ is due to $\mathrm{C}_{8}-\mathrm{H}$ and $-\mathrm{OH}$ proton was seen as a broad singlet around $\delta 11.40$, which was confirmed as stated earlier. The remaining aromatic protons appeared as expected. The ${ }^{13} \mathrm{C}-\mathrm{NMR}$ spectra of compounds $\mathbf{2 k - 0}$ showed signals around $\delta$ 28.5, 33.6, 44.7 and 54.5 due to two methyl group attached at $\mathrm{C}_{10}, \mathrm{C}_{10}$ (itself), $\mathrm{C}_{9}$ and $\mathrm{C}_{11}$ respectively. This was further confirmed by DEPT-135 spectra in which $\mathrm{C}_{9}$ and $\mathrm{C}_{11}$ signals got inverted. The incorporation of naphthalenone ring in the compounds $\mathbf{2 k - \mathbf { o }}$ is this supported. The carbonyl carbon signals in naphthalenone ring and $\delta$-lactone ring appeared around $\delta 200.0$ and $\delta$ 163.0 respectively. The aromatic carbons appeared between $\delta 108.0$ and 162.0. The signal around $\delta 162.0$ is due to $\mathrm{C}_{7}-\mathrm{OH}$. Mass spectra of compound $2 \mathbf{k}$ gave molecular ion peak at 308.1 $\left(\mathrm{M}^{+}\right)$corresponding to molecular formula $\mathrm{C}_{19} \mathrm{H}_{16} \mathrm{O}_{4}$.

All other compounds gave satisfactory spectral data which are given in experimental section. 


\section{Conclusions}

In summary, a simple, convenient and general method has been developed for the preparation of fused chromenes utilizing easily accessible and inexpensive starting materials. This synthetic approach includes some important aspects such as high yields and mild reaction conditions, which make this synthetic protocol a useful and an attractive procedure for the synthesis of indanone and naphthalenone fused chromenones derivatives. This reaction can be regarded as a new approach for the preparation of synthetically and pharmaceutically relevant heterocyclic systems.

\section{Experimental Section}

General. Reagents and solvents were obtaind from commercial sources and used without further purification. All melting points were taken in open capillaries and are uncorrected. Thin-layer chromatography (TLC, on aluminum plates coated with silica gel $60 \mathrm{~F}_{254}, 0.25 \mathrm{~mm}$ thickness, Merck) was used for monitoring the progress of all reactions, purity and homogeneity of the synthesized compounds. Elemental analysis (\% C, H, N) was carried out by Perkin-Elmer 2400 series-II elemental analyzer at Sophisticated Instrumentation Centre for Applied Research \& Training (SICART), Vallabh Vidhyanagar and result obtained for those elements are within $\pm 0.4 \%$ of the theoretical values. The FTIR spectra were recorded using potassium bromide disc on a Shimadzu FTIR 8401 spectrophotometer and only the characteristic peaks are reported. ${ }^{1} \mathrm{H}-$ NMR and ${ }^{13} \mathrm{C}$-NMR spectra were recorded using DMSO- $d_{6}$ solvent on a Bruker Avance 400 $(\mathrm{MHz})$ spectrometer using solvent peak as internal standard at 400 and $100 \mathrm{MHz}$, respectively. Chemical shifts are reported in parts per million ( $\mathrm{ppm})$. Mass spectra were scanned on a Shimadzu LCMS 2010 spectrometer. 1-(2-oxo-2-(2-oxo-2H-chromen-3-yl) ethyl) pyridinium bromide $\mathbf{1}(\mathbf{a}-\mathbf{e})$ was prepared according the literature procedures ${ }^{21}$.

General procedure for the synthesis of fused chromenones (2a-0). In a round bottom flask $(100 \mathrm{~mL})$, a solution of appropriate diketone (1,3-cyclopentandione or 1,3-cyclohexandione or dimedone) $(0.0058 \mathrm{~mol})$ was taken in glacial acetic acid $(15 \mathrm{~mL})$. To this solution, sodium acetate $(0.06 \mathrm{~mol})$ and an appropriate 1-(2-oxo-2-(2-oxo- $2 \mathrm{H}$-chromen-3-yl) ethyl) pyridinium bromide 1a-e $(0.006 \mathrm{~mol})$ in acetic acid $(10 \mathrm{~mL})$ were added with stirring. The reaction mixture was stirred at room temperature for 45 minutes and then refluxed in an oil bath at $140-145^{\circ} \mathrm{C}$ for 6 hours and left overnight. It was then poured in water $(75 \mathrm{~mL})$ and the crude solid obtained was extracted with chloroform $(3 \times 50 \mathrm{~mL})$. The organic layer was washed with $10 \%$ sodium bicarbonate solution $(50 \mathrm{~mL})$, water $(50 \mathrm{~mL})$ and dried over anhydrous sodium sulfate. Distillation of chloroform in vacuum gave gummy material which was subjected to column chromatography using ethyl acetate-pet.ether (60-80) (2:8) as an eluent to afford product 2a-o respectively. The product was recrystallized from chloroform-hexane. 
7-Hydroxy-9,10-dihydroindeno[5,4-c]chromene-6,11-dione (2a). Yield 62\%; mp 222-224 ${ }^{\circ} \mathrm{C}$; white crystalline solid; Selected IR frequencies (KBr): 3445 (broad, -OH), 2926 (C-H, aliphatic), $1670(\mathrm{C}=\mathrm{O}, \delta$-lactone $), 1710(\mathrm{C}=\mathrm{O}$, indanone $), 1615(\mathrm{C}=\mathrm{C}$, aromatic $) \mathrm{cm}^{-1} ;{ }^{1} \mathrm{H} \mathrm{NMR}\left(\mathrm{CDCl}_{3}\right): \delta$ $3.08\left(2 \mathrm{H}, \mathrm{t}, \mathrm{C}_{9}-\mathrm{H}, J 6.8 \mathrm{~Hz}\right), 3.15\left(2 \mathrm{H}, \mathrm{t}, \mathrm{C}_{10}-\mathrm{H}, J 6.8 \mathrm{~Hz}\right), 7.33-7.61(4 \mathrm{H}, \mathrm{m}, \mathrm{Ar}-\mathrm{H}), 8.07(1 \mathrm{H}, \mathrm{s}$, $\left.\mathrm{C}_{8}-\mathrm{H}\right), 11.30\left(1 \mathrm{H}, \mathrm{s},-\mathrm{OH}\right.$ proton, $\mathrm{D}_{2} \mathrm{O}$ exchangeable); ${ }^{13} \mathrm{C}$ NMR: $\delta 28.0\left(\mathrm{CH}_{2}\right), 29.0\left(\mathrm{CH}_{2}\right), 105.0$ $(\mathrm{C}), 114.7(\mathrm{CH}), 117.8(\mathrm{CH}), 118.4(\mathrm{C}), 123.3(\mathrm{CH}), 127.2(\mathrm{CH}), 129.8(\mathrm{C}), 130.6(\mathrm{CH}), 136.5$ (C), $139.2(\mathrm{C}), 150.2(\mathrm{C}), 161.6\left(\mathrm{C}_{7}\right), 163.0(\mathrm{C}=\mathrm{O}, \delta$-lactone $), 200.0(\mathrm{C}=\mathrm{O}$, indanone); Anal. Calcd. for $\mathrm{C}_{16} \mathrm{H}_{10} \mathrm{O}_{4}$ : C, 72.18; H, 3.79\%. Found: C, 72.08; H, 3.51\%. MS: $266.0\left(\mathrm{M}^{+}\right)$.

3,7-Dihydroxy-9,10-dihydroindeno[5,4-c]chromene-6,11-dione (2b). Yield 57\%; mp 176-178 ${ }^{\circ} \mathrm{C}$; white crystalline solid; Selected IR frequencies $(\mathrm{KBr})$ : 3442 (broad, -OH), $2935(\mathrm{C}-\mathrm{H}$, aliphatic), $1680(\mathrm{C}=\mathrm{O}, \delta$-lactone $), 1720\left(\mathrm{C}=\mathrm{O}\right.$, indanone), $1610(\mathrm{C}=\mathrm{C}$, aromatic $) \mathrm{cm}^{-1} ;{ }^{1} \mathrm{H}$ NMR $\left(\mathrm{CDCl}_{3}\right): \delta 3.05\left(2 \mathrm{H}, \mathrm{t}, \mathrm{C}_{9}-\mathrm{H}, J 6.7 \mathrm{~Hz}\right), 3.13\left(2 \mathrm{H}, \mathrm{t}, \mathrm{C}_{10}-\mathrm{H}, J 6.7 \mathrm{~Hz}\right), 7.34-7.59(3 \mathrm{H}, \mathrm{m}, \mathrm{Ar}-\mathrm{H})$, $8.09\left(1 \mathrm{H}, \mathrm{s}, \mathrm{C}_{8}-\mathrm{H}\right), 11.30\left(1 \mathrm{H}, \mathrm{s},-\mathrm{OH}\right.$ proton, $\mathrm{D}_{2} \mathrm{O}$ exchangeable $), 11.83(1 \mathrm{H}, \mathrm{s},-\mathrm{OH}$ proton, $\mathrm{D}_{2} \mathrm{O}$ exchangeable); ${ }^{13} \mathrm{C}$ NMR: $\delta 28.5\left(\mathrm{CH}_{2}\right), 29.8\left(\mathrm{CH}_{2}\right), 105.7(\mathrm{C}), 113.2(\mathrm{CH}), 116.7(\mathrm{CH})$, $118.7(\mathrm{C}), 123.4(\mathrm{CH}), 127.5(\mathrm{CH}), 129.8(\mathrm{C}), 135.7(\mathrm{C}), 140.3(\mathrm{C}), 150.2(\mathrm{C}), 159.7(\mathrm{C}), 160.7$ $\left(\mathrm{C}_{7}\right), 163.3\left(\mathrm{C}=\mathrm{O}, \delta\right.$-lactone), $199.9\left(\mathrm{C}=\mathrm{O}\right.$, indanone); Anal. Calcd. for $\mathrm{C}_{16} \mathrm{H}_{10} \mathrm{O}_{5}: \mathrm{C}, 68.09 ; \mathrm{H}$, $3.57 \%$. Found: $\mathrm{C}, 68.23 ; \mathrm{H}, 3.42 \%$.

7-Hydroxy-3-methoxy-9,10-dihydroindeno[5,4-c]chromene-6,11-dione (2c). Yield 63\%; mp 228-230 ${ }^{\circ} \mathrm{C}$; off-white solid; Selected IR frequencies (KBr): 3439 (broad, -OH), $2948(\mathrm{C}-\mathrm{H}$, aliphatic $), 1670(\mathrm{C}=\mathrm{O}, \delta$-lactone $), 1730(\mathrm{C}=\mathrm{O}$, indanone $), 1622(\mathrm{C}=\mathrm{C}$, aromatic $), 1055$ (asymmetric and symmetric C-O-C stretching) $\mathrm{cm}^{-1} ;{ }^{1} \mathrm{H}$ NMR $\left(\mathrm{CDCl}_{3}\right): \delta 3.07(2 \mathrm{H}, \mathrm{t}, \mathrm{C} 9-\mathrm{H}, J 6.8$ $\mathrm{Hz}), 3.11\left(2 \mathrm{H}, \mathrm{t}, \mathrm{C}_{10}-\mathrm{H}, J 6.8 \mathrm{~Hz}\right), 3.94\left(3 \mathrm{H}, \mathrm{s}, \mathrm{OCH}_{3}\right), 7.32-7.55(3 \mathrm{H}, \mathrm{m}, \mathrm{Ar}-\mathrm{H}), 8.11(1 \mathrm{H}, \mathrm{s}$, $\left.\mathrm{C}_{8}-\mathrm{H}\right), 11.42\left(1 \mathrm{H}, \mathrm{s}\right.$, -OH proton, $\mathrm{D}_{2} \mathrm{O}$ exchangeable); ${ }^{13} \mathrm{C}$ NMR: $\delta 28.4\left(\mathrm{CH}_{2}\right), 28.8\left(\mathrm{CH}_{2}\right), 56.2$ $\left(\mathrm{OCH}_{3}\right), 106.7(\mathrm{C}), 114.1(\mathrm{CH}), 116.4(\mathrm{CH}), 119.5(\mathrm{C}), 122.2(\mathrm{CH}), 126.4(\mathrm{CH}), 129.2(\mathrm{C})$, $136.8(\mathrm{C}), 140.1(\mathrm{C}), 150.4(\mathrm{C}), 158.8(\mathrm{C}), 161.7\left(\mathrm{C}_{7}\right), 163.5$ (C=O, $\delta$-lactone), $200.1(\mathrm{C}=\mathrm{O}$, indanone); Anal. Calcd. for $\mathrm{C}_{17} \mathrm{H}_{12} \mathrm{O}_{5}: \mathrm{C}, 68.92 ; \mathrm{H}, 4.08 \%$. Found: $\mathrm{C}, 68.83 ; \mathrm{H}, 3.97 \%$.

4,7-Dihydroxy-9,10-dihydroindeno[5,4-c] chromene-6,11-dione (2d). Yield 64\%; mp 195-198 ${ }^{\circ} \mathrm{C}$; white crystalline solid; Selected IR frequencies $(\mathrm{KBr}): 3437$ (broad, -OH), $2933(\mathrm{C}-\mathrm{H}$, aliphatic), $1680(\mathrm{C}=\mathrm{O}, \delta$-lactone $), 1715(\mathrm{C}=\mathrm{O}$, indanone $), 1617(\mathrm{C}=\mathrm{C}$, aromatic $) \mathrm{cm}^{-1} ;{ }^{1} \mathrm{H}$ NMR $\left(\mathrm{CDCl}_{3}\right): \delta 3.08(2 \mathrm{H}, \mathrm{t}, \mathrm{C} 9-\mathrm{H}, J 6.7 \mathrm{~Hz}), 3.10\left(2 \mathrm{H}, \mathrm{t}, \mathrm{C}_{10}-\mathrm{H}, J 6.7 \mathrm{~Hz}\right), 7.30-7.57(3 \mathrm{H}, \mathrm{m}, \mathrm{Ar}-\mathrm{H})$, $8.08\left(1 \mathrm{H}, \mathrm{s}, \mathrm{C}_{8}-\mathrm{H}\right), 11.35\left(1 \mathrm{H}, \mathrm{s},-\mathrm{OH}\right.$ proton, $\mathrm{D}_{2} \mathrm{O}$ exchangeable $), 11.78(1 \mathrm{H}, \mathrm{s},-\mathrm{OH}$ proton, $\mathrm{D}_{2} \mathrm{O}$ exchangeable); ${ }^{13} \mathrm{C}$ NMR: $\delta 28.8\left(\mathrm{CH}_{2}\right), 29.4\left(\mathrm{CH}_{2}\right), 105.2(\mathrm{C}), 113.2(\mathrm{CH}), 116.9(\mathrm{CH})$, $118.7(\mathrm{C}), 122.1(\mathrm{CH}), 127.8(\mathrm{CH}), 130.2(\mathrm{C}), 135.2(\mathrm{C}), 140.5(\mathrm{C}), 151.8(\mathrm{C}), 159.1(\mathrm{C}), 160.1$ $\left(\mathrm{C}_{7}\right), 163.8(\mathrm{C}=\mathrm{O}, \delta$-lactone $), 200.0\left(\mathrm{C}=\mathrm{O}\right.$, indanone); Anal. Calcd. for $\mathrm{C}_{16} \mathrm{H}_{10} \mathrm{O}_{5}: \mathrm{C}, 68.09 ; \mathrm{H}$, $3.57 \%$. Found: C, $68.11 ; \mathrm{H}, 3.71 \%$.

7-Hydroxy-4-methoxy-9,10-dihydroindeno[5,4-c]chromene-6,11-dione (2e). Yield 67\%; mp 173-176 ${ }^{\circ} \mathrm{C}$; off-white solid; Selected IR frequencies (KBr): 3445 (broad, -OH), 2933 (C-H, aliphatic $), 1670(\mathrm{C}=\mathrm{O}, \delta$-lactone $), 1720(\mathrm{C}=\mathrm{O}$, indanone $), 1621(\mathrm{C}=\mathrm{C}$, aromatic $), 1050$ (asymmetric and symmetric C-O-C stretching) $\mathrm{cm}^{-1} ;{ }^{1} \mathrm{H}$ NMR $\left(\mathrm{CDCl}_{3}\right): \delta 3.03(2 \mathrm{H}, \mathrm{t}, \mathrm{C} 9-\mathrm{H}, J 6.8$ $\mathrm{Hz}), 3.13\left(2 \mathrm{H}, \mathrm{t}, \mathrm{C}_{10}-\mathrm{H}, J 6.8 \mathrm{~Hz}\right), 3.97\left(3 \mathrm{H}, \mathrm{s}, \mathrm{OCH}_{3}\right), 7.29-7.49(3 \mathrm{H}, \mathrm{m}, \mathrm{Ar}-\mathrm{H}), 8.13(1 \mathrm{H}, \mathrm{s}$, 
$\left.\mathrm{C}_{8}-\mathrm{H}\right), 11.44\left(1 \mathrm{H}, \mathrm{s},-\mathrm{OH}\right.$ proton, $\mathrm{D}_{2} \mathrm{O}$ exchangeable); ${ }^{13} \mathrm{C} \mathrm{NMR}: \delta 28.2\left(\mathrm{CH}_{2}\right), 28.6\left(\mathrm{CH}_{2}\right), 56.24$ $\left(\mathrm{OCH}_{3}\right), 105.9(\mathrm{C}), 114.3(\mathrm{CH}), 117.1(\mathrm{CH}), 120.3(\mathrm{C}), 122.2(\mathrm{CH}), 126.7(\mathrm{CH}), 128.9(\mathrm{C})$, 136.9 (C), 139.9 (C), $150.7(\mathrm{C}), 152.7(\mathrm{C}), 162.3\left(\mathrm{C}_{7}\right), 163.0(\mathrm{C}=\mathrm{O}, \delta$-lactone), $200.2(\mathrm{C}=\mathrm{O}$, indanone); Anal. Calcd. for $\mathrm{C}_{17} \mathrm{H}_{12} \mathrm{O}_{5}$ : C, 68.92; H, 4.08\%. Found: C, 68.89; H, 4.11\%.

7-Hydroxy-10,11-dihydro-6H-naphtho[2,1-c]chromene-6,12(9H)-dione (2f). Yield 61\%; mp 180-182 ${ }^{\circ} \mathrm{C}$; white solid; Selected IR frequencies (KBr): 3430 (broad, -OH), 2900 (C-H, aliphatic), $1665(\mathrm{C}=\mathrm{O}, \delta$-lactone $), 1720(\mathrm{C}=\mathrm{O}$, naphthalenone $), 1610(\mathrm{C}=\mathrm{C}$, aromatic $) \mathrm{cm}^{-1} ;{ }^{1} \mathrm{H}$ NMR $\left(\mathrm{CDCl}_{3}\right): \delta 2.25\left(2 \mathrm{H}, \mathrm{m}, \mathrm{C}_{10}-\mathrm{H}\right), 2.76\left(2 \mathrm{H}, \mathrm{t}, \mathrm{C}_{9}-\mathrm{H}, J 5.5 \mathrm{~Hz}\right), 2.95\left(2 \mathrm{H}, \mathrm{t}, \mathrm{C}_{11}-\mathrm{H}, J 5.5 \mathrm{~Hz}\right)$, 7.33-7.62 (4H, m, Ar-H), $8.15\left(1 \mathrm{H}, \mathrm{s}, \mathrm{C}_{8}-\mathrm{H}\right), 11.42\left(1 \mathrm{H}, \mathrm{s},-\mathrm{OH}\right.$ proton, $\mathrm{D}_{2} \mathrm{O}$ exchangeable $) ;{ }^{13} \mathrm{C}$ NMR: $\delta 23.8\left(\mathrm{CH}_{2}\right), 30.2\left(\mathrm{CH}_{2}\right), 35.6\left(\mathrm{CH}_{2}\right), 107.9(\mathrm{C}), 111.3(\mathrm{C}), 116.3(\mathrm{CH}), 120.7(\mathrm{CH})$, $122.6(\mathrm{CH}), 125.7(\mathrm{C}), 128.3(\mathrm{CH}), 135.4(\mathrm{CH}), 140.1(\mathrm{C}), 149.5(\mathrm{C}), 158.7(\mathrm{C}), 161.3\left(\mathrm{C}_{7}\right)$, $163.2\left(\mathrm{C}=\mathrm{O}, \delta\right.$-lactone), $197.2\left(\mathrm{C}=\mathrm{O}\right.$, naphthalenone); Anal. Calcd. for $\mathrm{C}_{17} \mathrm{H}_{12} \mathrm{O}_{4}$ : $\mathrm{C}, 72.85 ; \mathrm{H}$, 4.32\%. Found: C, 72.71; H, 4.25\%. MS: $280.0\left(\mathrm{M}^{+}\right)$.

3,7-Dihydroxy-10,11-dihydro-6H-naphtho[2,1-c]chromene-6,12(9H)-dione (2g). Yield 69\%; mp 210-213 ${ }^{\circ} \mathrm{C}$; white solid; Selected IR frequencies (KBr): 3440 (broad, -OH), 2930 (C-H, aliphatic), $1660\left(\mathrm{C}=\mathrm{O}, \delta\right.$-lactone), $1710(\mathrm{C}=\mathrm{O}$, naphthalenone $), 1615(\mathrm{C}=\mathrm{C}$, aromatic $) \mathrm{cm}^{-1} ;{ }^{1} \mathrm{H}$ NMR $\left(\mathrm{CDCl}_{3}\right): \delta 2.20\left(2 \mathrm{H}, \mathrm{m}, \mathrm{C}_{10}-\mathrm{H}\right), 2.71\left(2 \mathrm{H}, \mathrm{t}, \mathrm{C}_{9}-\mathrm{H}, J 5.5 \mathrm{~Hz}\right), 2.93\left(2 \mathrm{H}, \mathrm{t}, \mathrm{C}_{11}-\mathrm{H}, J 5.5 \mathrm{~Hz}\right)$, 7.34-7.59 (3H, m, Ar-H), $8.09\left(1 \mathrm{H}, \mathrm{s}, \mathrm{C}_{8}-\mathrm{H}\right), 11.39$ (1H, s, -OH proton, $\mathrm{D}_{2} \mathrm{O}$ exchangeable), $11.83\left(1 \mathrm{H}, \mathrm{s},-\mathrm{OH}\right.$ proton, $\mathrm{D}_{2} \mathrm{O}$ exchangeable); ${ }^{13} \mathrm{C}$ NMR: $\delta 23.3\left(\mathrm{CH}_{2}\right), 29.8\left(\mathrm{CH}_{2}\right), 34.8\left(\mathrm{CH}_{2}\right)$, $107.3(\mathrm{C}), 111.7(\mathrm{C}), 116.8(\mathrm{CH}), 121.3(\mathrm{CH}), 121.8(\mathrm{CH}), 125.5(\mathrm{C}), 128.2(\mathrm{C}), 134.9(\mathrm{CH})$, 140.8 (C), 151.4 (C), 158.9 (C), $161.7 \quad\left(\mathrm{C}_{7}\right), 163.8$ (C=O, $\delta$-lactone), 197.8 (C=O, naphthalenone); Anal. Calcd. for $\mathrm{C}_{17} \mathrm{H}_{12} \mathrm{O}_{5}$ : C, 68.92; H, 4.08\%;. Found: C, 68.79; H, 4.18\%.

7-Hydroxy-3-methoxy-10,11-dihydro-6H-naphtho[2,1-c]chromene-6,12(9H)-dione (2h). Yield 64\%; mp 190-194 ${ }^{\circ} \mathrm{C}$; off-white solid; Selected IR frequencies (KBr): 3435 (broad, -OH), 2940 (C-H, aliphatic), 1670 ( $\mathrm{C}=\mathrm{O}, \delta$-lactone), 1730 (C=O, naphthalenone), 1622 (C=C, aromatic), 1050 (asymmetric and symmetric $\mathrm{C}-\mathrm{O}-\mathrm{C}$ stretching) $\mathrm{cm}^{-1} ;{ }^{1} \mathrm{H} \mathrm{NMR}\left(\mathrm{CDCl}_{3}\right): \delta 2.27$ $\left(2 \mathrm{H}, \mathrm{m}, \mathrm{C}_{10}-\mathrm{H}\right), 2.73\left(2 \mathrm{H}, \mathrm{t}, \mathrm{C}_{9}-\mathrm{H}, J 5.5 \mathrm{~Hz}\right), 2.97\left(2 \mathrm{H}, \mathrm{t}, \mathrm{C}_{11}-\mathrm{H}, J 5.5 \mathrm{~Hz}\right), 3.40\left(3 \mathrm{H}, \mathrm{s}, \mathrm{OCH}_{3}\right)$, 7.31-7.60 (3H, m, Ar-H), $8.08\left(1 \mathrm{H}, \mathrm{s}, \mathrm{C}_{8}-\mathrm{H}\right), 11.44\left(1 \mathrm{H}, \mathrm{s},-\mathrm{OH}\right.$ proton, $\mathrm{D}_{2} \mathrm{O}$ exchangeable $) ;{ }^{13} \mathrm{C}$ NMR: $\delta 23.4\left(\mathrm{CH}_{2}\right), 30.1\left(\mathrm{CH}_{2}\right), 35.2\left(\mathrm{CH}_{2}\right), 56.4\left(\mathrm{OCH}_{3}\right), 107.8(\mathrm{C}), 111.5(\mathrm{C}), 116.7(\mathrm{CH})$, $120.9(\mathrm{CH}), 122.3(\mathrm{CH}), 125.4(\mathrm{C}), 128.4(\mathrm{C}), 135.7(\mathrm{CH}), 141.2(\mathrm{C}), 151.5(\mathrm{C}), 157.8(\mathrm{C})$, $161.9\left(\mathrm{C}_{7}\right), 162.8(\mathrm{C}=\mathrm{O}, \delta$-lactone $), 197.7\left(\mathrm{C}=\mathrm{O}\right.$, naphthalenone); Anal. Calcd. for $\mathrm{C}_{18} \mathrm{H}_{14} \mathrm{O}_{5}: \mathrm{C}$, 69.67; H, 4.55\%. Found: C, 69.79; H, 4.35\%.

4,7-Dihydroxy-10,11-dihydro-6H-naphtho[2,1-c]chromene-6,12(9H)-dione (2i). Yield 67\%; mp 203-207 ${ }^{\circ} \mathrm{C}$; white crystalline solid; Selected IR frequencies (KBr): 3440 (broad, -OH), 2925 (C-H, aliphatic), $1650(\mathrm{C}=\mathrm{O}, \delta$-lactone $), 1715(\mathrm{C}=\mathrm{O}$, naphthalenone $), 1610(\mathrm{C}=\mathrm{C}$, aromatic $) \mathrm{cm}^{-}$ 1; ${ }^{1} \mathrm{H} \mathrm{NMR}\left(\mathrm{CDCl}_{3}\right): \delta 2.22\left(2 \mathrm{H}, \mathrm{m}, \mathrm{C}_{10}-\mathrm{H}\right), 2.77\left(2 \mathrm{H}, \mathrm{t}, \mathrm{C}_{9}-\mathrm{H}, J 5.5 \mathrm{~Hz}\right), 2.95\left(2 \mathrm{H}, \mathrm{t}, \mathrm{C}_{11}-\mathrm{H}, J\right.$ $5.5 \mathrm{~Hz}), 7.33-7.61(3 \mathrm{H}, \mathrm{m}, \mathrm{Ar}-\mathrm{H}), 8.11\left(1 \mathrm{H}, \mathrm{s}, \mathrm{C}_{8}-\mathrm{H}\right), 11.42\left(1 \mathrm{H}, \mathrm{s},-\mathrm{OH}\right.$ proton, $\mathrm{D}_{2} \mathrm{O}$ exchangeable), $11.86\left(1 \mathrm{H}, \mathrm{s},-\mathrm{OH}\right.$ proton, $\mathrm{D}_{2} \mathrm{O}$ exchangeable); ${ }^{13} \mathrm{C} \mathrm{NMR}: \delta 22.9\left(\mathrm{CH}_{2}\right), 30.2$ $\left(\mathrm{CH}_{2}\right), 35.1\left(\mathrm{CH}_{2}\right), 107.2(\mathrm{C}), 111.8(\mathrm{C}), 116.5(\mathrm{CH}), 120.8(\mathrm{CH}), 121.6(\mathrm{CH}), 125.3(\mathrm{C}), 128.4$ (C), $135.1(\mathrm{CH}), 141.1(\mathrm{C}), 151.6(\mathrm{C}), 158.7(\mathrm{C}), 161.2\left(\mathrm{C}_{7}\right), 163.5(\mathrm{C}=\mathrm{O}, \delta$-lactone $), 197.2$ 
( $\mathrm{C}=\mathrm{O}$, naphthalenone); Anal. Calcd. for $\mathrm{C}_{17} \mathrm{H}_{12} \mathrm{O}_{5}$ : C, 68.92; H, 4.08\%;. Found: C, 68.75; H, $3.98 \%$.

7-Hydroxy-4-methoxy-10,11-dihydro-6H-naphtho[2,1-c]chromene-6,12(9H)-dione $\quad(2 \mathbf{j})$. Yield 61\%; mp 183-186 ${ }^{\circ} \mathrm{C}$; off-white solid; Selected IR frequencies (KBr): 3450 (broad, $-\mathrm{OH}$ ), 2930 (C-H, aliphatic), 1670 ( $\mathrm{C}=\mathrm{O}, \delta$-lactone), 1720 ( $\mathrm{C}=\mathrm{O}$, indanone), 1621 ( $\mathrm{C}=\mathrm{C}$, aromatic), 1055 (asymmetric and symmetric C-O-C stretching) $\mathrm{cm}^{-1} ;{ }^{1} \mathrm{H} \mathrm{NMR}\left(\mathrm{CDCl}_{3}\right): \delta 2.20\left(2 \mathrm{H}, \mathrm{m}, \mathrm{C}_{10^{-}}\right.$ H), $2.70\left(2 \mathrm{H}, \mathrm{t}, \mathrm{C}_{9}-\mathrm{H}, J 5.5 \mathrm{~Hz}\right), 2.96\left(2 \mathrm{H}, \mathrm{t}, \mathrm{C}_{11}-\mathrm{H}, J 5.5 \mathrm{~Hz}\right), 3.97\left(3 \mathrm{H}, \mathrm{s}, \mathrm{OCH}_{3}\right), 7.28-7.67$ $(3 \mathrm{H}, \mathrm{m}, \mathrm{Ar}-\mathrm{H}), 8.15\left(1 \mathrm{H}, \mathrm{s}, \mathrm{C}_{8}-\mathrm{H}\right), 11.40\left(1 \mathrm{H}, \mathrm{s},-\mathrm{OH}\right.$ proton, $\mathrm{D}_{2} \mathrm{O}$ exchangeable $) ;{ }^{13} \mathrm{C}$ NMR: $\delta$ $23.3\left(\mathrm{CH}_{2}\right), 30.4\left(\mathrm{CH}_{2}\right), 34.9\left(\mathrm{CH}_{2}\right), 56.3\left(\mathrm{OCH}_{3}\right), 107.9(\mathrm{C}), 111.8(\mathrm{C}), 116.5(\mathrm{CH}), 121.2(\mathrm{CH})$, $122.4(\mathrm{CH}), 125.3(\mathrm{C}), 128.7(\mathrm{C}), 136.2(\mathrm{CH}), 140.9(\mathrm{C}), 151.4(\mathrm{C}), 157.8(\mathrm{C}), 161.5\left(\mathrm{C}_{7}\right), 163.1$ $\left(\mathrm{C}=\mathrm{O}, \delta\right.$-lactone), $197.8\left(\mathrm{C}=\mathrm{O}\right.$, naphthalenone); Anal. Calcd. for $\mathrm{C}_{18} \mathrm{H}_{14} \mathrm{O}_{5}: \mathrm{C}, 69.67 ; \mathrm{H}, 4.55 \%$. Found: C, 69.55; H, $4.72 \%$.

7-Hydroxy-10,10-dimethyl-10,11-dihydro-6H-naphtho[2,1-c]chromene-6,12(9H)-dione (2k). Yield 66\%; mp 180-182 ${ }^{\circ} \mathrm{C}$; off-white solid; Selected IR frequencies (KBr): 3435 (broad, -OH), 3020 (C-H, aliphatic), 1660 (C=O, $\delta$-lactone), $1715(\mathrm{C}=\mathrm{O}$, naphthalenone), $1605(\mathrm{C}=\mathrm{C}$, aromatic) $\mathrm{cm}^{-1} ;{ }^{1} \mathrm{H}$ NMR $\left(\mathrm{CDCl}_{3}\right): \delta 1.11\left(6 \mathrm{H}, \mathrm{s}, 2 \times \mathrm{CH}_{3}\right), 2.70(2 \mathrm{H}, \mathrm{s}, \mathrm{C} 9-\mathrm{H}), 2.95\left(2 \mathrm{H}, \mathrm{s}, \mathrm{C}_{11^{-}}\right.$ $\mathrm{H})$, 7.30-7.59 (4H, m, Ar-H), $8.20\left(1 \mathrm{H}, \mathrm{s}, \mathrm{C}_{8}-\mathrm{H}\right), 11.44\left(1 \mathrm{H}, \mathrm{s},-\mathrm{OH}\right.$ proton, $\mathrm{D}_{2} \mathrm{O}$ exchangeable); ${ }^{13} \mathrm{C}$ NMR: $\delta 28.2\left(2 \times \mathrm{CH}_{3}\right), 33.6(\mathrm{C}), 44.7\left(\mathrm{CH}_{2}\right), 54.5\left(\mathrm{CH}_{2}\right), 108.6(\mathrm{C}), 112.4(\mathrm{C}), 117.5(\mathrm{CH})$, $121.5(\mathrm{CH}), 123.6(\mathrm{CH}), 127.2(\mathrm{C}), 128.4(\mathrm{CH}), 137.3(\mathrm{CH}), 142.3(\mathrm{C}), 150.5(\mathrm{C}), 158.8(\mathrm{C})$, $162.3\left(\mathrm{C}_{7}\right), 163.3\left(\mathrm{C}=\mathrm{O}, \delta\right.$-lactone), $200.9\left(\mathrm{C}=\mathrm{O}\right.$, naphthalenone); Anal. Calcd. for $\mathrm{C}_{19} \mathrm{H}_{16} \mathrm{O}_{4}: \mathrm{C}$, 74.01; H, 5.23\%. Found: C, 74.21; H, 5.33\%. MS: 308.1 $\left(\mathrm{M}^{+}\right)$.

3,7-Dihydroxy-10,10-dimethyl-10,11-dihydro-6H-naphtho[2,1-c]chromene-6,12(9H)-dione

(2l). Yield 63\%; mp 207-210 ${ }^{\circ} \mathrm{C}$; white solid; Selected IR frequencies (KBr): 3440 (broad, $-\mathrm{OH}$ ), $3030(\mathrm{C}-\mathrm{H}$, aliphatic), $1650(\mathrm{C}=\mathrm{O}, \delta$-lactone $), 1710(\mathrm{C}=\mathrm{O}$, naphthalenone $), 1615(\mathrm{C}=\mathrm{C}$, aromatic) $\mathrm{cm}^{-1} ;{ }^{1} \mathrm{H} \mathrm{NMR}\left(\mathrm{CDCl}_{3}\right): \delta 1.11\left(6 \mathrm{H}, \mathrm{s}, 2 \times \mathrm{CH}_{3}\right), 2.73(2 \mathrm{H}, \mathrm{s}, \mathrm{C} 9-\mathrm{H}), 2.90\left(2 \mathrm{H}, \mathrm{s}, \mathrm{C}_{11^{-}}\right.$ $\mathrm{H})$, 7.34-7.61 (3H, m, Ar-H), $8.18\left(1 \mathrm{H}, \mathrm{s}, \mathrm{C}_{8}-\mathrm{H}\right), 11.39$ (1H, s, -OH proton, $\mathrm{D}_{2} \mathrm{O}$ exchangeable), $11.81\left(1 \mathrm{H}, \mathrm{s},-\mathrm{OH}\right.$ proton, $\mathrm{D}_{2} \mathrm{O}$ exchangeable); ${ }^{13} \mathrm{C} \mathrm{NMR}: \delta 28.3\left(2 \times \mathrm{CH}_{3}\right), 33.4(\mathrm{C}), 44.5\left(\mathrm{CH}_{2}\right)$, $54.2\left(\mathrm{CH}_{2}\right), 108.9(\mathrm{C}), 113.5(\mathrm{C}), 117.3(\mathrm{CH}), 121.7(\mathrm{CH}), 124.2(\mathrm{C}), 127.6(\mathrm{CH}), 128.9(\mathrm{C})$, $137.6(\mathrm{CH}), 143.5(\mathrm{C}), 151.2(\mathrm{C}), 158.4(\mathrm{C}), 162.7\left(\mathrm{C}_{7}\right), 163.8(\mathrm{C}=\mathrm{O}, \delta$-lactone $), 200.7(\mathrm{C}=\mathrm{O}$, naphthalenone); Anal. Calcd. for $\mathrm{C}_{19} \mathrm{H}_{16} \mathrm{O}_{5}$ : C, 70.36; H, 4.97\%. Found: C, 70.58; H, 5.20\%.

\section{7-Hydroxy-3-methoxy-10,10-dimethyl-10,11-dihydro-6H-naphtho[2,1-c]chromene-}

6,12(9H)-dione (2m). Yield 59\%; mp 182-186 ${ }^{\circ} \mathrm{C}$; white solid; Selected IR frequencies (KBr): 3435 (broad, -OH), 2990 ( $\mathrm{C}-\mathrm{H}$, aliphatic), 1670 ( $\mathrm{C}=\mathrm{O}, \delta$-lactone), 1730 ( $\mathrm{C}=\mathrm{O}$, naphthalenone), 1607 ( $\mathrm{C}=\mathrm{C}$, aromatic), 1055 (asymmetric and symmetric $\mathrm{C}-\mathrm{O}-\mathrm{C}$ stretching) $\mathrm{cm}^{-1}$; ${ }^{1} \mathrm{H} \mathrm{NMR}$ $\left(\mathrm{CDCl}_{3}\right): \delta 1.11\left(6 \mathrm{H}, \mathrm{s}, 2 \times \mathrm{CH}_{3}\right), 2.72(2 \mathrm{H}, \mathrm{s}, \mathrm{C}-\mathrm{H}), 2.96\left(2 \mathrm{H}, \mathrm{s}, \mathrm{C}_{11}-\mathrm{H}\right), 3.39\left(3 \mathrm{H}, \mathrm{s}, \mathrm{OCH}_{3}\right)$, 7.35-7.69 (3H, m, Ar-H), $8.16\left(1 \mathrm{H}, \mathrm{s}, \mathrm{C}_{8}-\mathrm{H}\right), 11.40\left(1 \mathrm{H}, \mathrm{s},-\mathrm{OH}\right.$ proton, $\mathrm{D}_{2} \mathrm{O}$ exchangeable $) ;{ }^{13} \mathrm{C}$ NMR: $\delta 28.4\left(2 \times \mathrm{CH}_{3}\right), 33.4(\mathrm{C}), 44.3\left(\mathrm{CH}_{2}\right), 54.4\left(\mathrm{CH}_{2}\right), 56.4\left(\mathrm{OCH}_{3}\right), 109.4(\mathrm{C}), 113.3(\mathrm{C})$, $116.7(\mathrm{CH}), 120.8(\mathrm{CH}), 124.3(\mathrm{CH}), 127.4(\mathrm{C}), 128.4(\mathrm{C}), 136.7(\mathrm{CH}), 143.2(\mathrm{C}), 151.3(\mathrm{C})$, $158.8(\mathrm{C}), 162.9\left(\mathrm{C}_{7}\right), 163.5(\mathrm{C}=\mathrm{O}, \delta$-lactone $), 200.3(\mathrm{C}=\mathrm{O}$, naphthalenone); Anal. Calcd. for $\mathrm{C}_{20} \mathrm{H}_{18} \mathrm{O}_{5}: \mathrm{C}, 70.99 ; \mathrm{H}, 5.36 \%$. Found: C, 70.87; H, 5.55\%. 


\section{4,7-Dihydroxy-10,10-dimethyl-10,11-dihydro-6H-naphtho[2,1-c]chromene-6,12(9H)-dione}

(2n). Yield 66\%; mp 201-204 ${ }^{\circ} \mathrm{C}$; white solid; Selected IR frequencies (KBr): 3440 (broad, $\mathrm{OH}), 3033(\mathrm{C}-\mathrm{H}$, aliphatic), $1650(\mathrm{C}=\mathrm{O}, \delta$-lactone $), 1710(\mathrm{C}=\mathrm{O}$, naphthalenone $), 1617(\mathrm{C}=\mathrm{C}$, aromatic) $\mathrm{cm}^{-1}$; ${ }^{1} \mathrm{H}$ NMR $\left(\mathrm{CDCl}_{3}\right): \delta 1.11\left(6 \mathrm{H}, \mathrm{s}, 2 \times \mathrm{CH}_{3}\right), 2.72(2 \mathrm{H}, \mathrm{s}, \mathrm{C} 9-\mathrm{H}), 2.92\left(2 \mathrm{H}, \mathrm{s}, \mathrm{C}_{11^{-}}\right.$ $\mathrm{H})$, 7.33-7.67 (3H, m, Ar-H), $8.15\left(1 \mathrm{H}, \mathrm{s}, \mathrm{C}_{8}-\mathrm{H}\right), 11.40\left(1 \mathrm{H}, \mathrm{s},-\mathrm{OH}\right.$ proton, $\mathrm{D}_{2} \mathrm{O}$ exchangeable), $11.82\left(1 \mathrm{H}, \mathrm{s},-\mathrm{OH}\right.$ proton, $\mathrm{D}_{2} \mathrm{O}$ exchangeable); ${ }^{13} \mathrm{C} \mathrm{NMR}: \delta 28.2\left(2 \times \mathrm{CH}_{3}\right), 33.7(\mathrm{C}), 44.7\left(\mathrm{CH}_{2}\right)$, $54.6\left(\mathrm{CH}_{2}\right), 109.2(\mathrm{C}), 113.2(\mathrm{C}), 117.8(\mathrm{CH}), 121.6(\mathrm{CH}), 123.8(\mathrm{C}), 127.9(\mathrm{CH}), 129.3(\mathrm{C})$, $137.2(\mathrm{CH}), 143.2(\mathrm{C}), 151.7(\mathrm{C}), 158.8(\mathrm{C}), 162.3\left(\mathrm{C}_{7}\right), 163.6(\mathrm{C}=\mathrm{O}, \delta$-lactone $), 200.1(\mathrm{C}=\mathrm{O}$, naphthalenone); Anal. Calcd. for $\mathrm{C}_{19} \mathrm{H}_{16} \mathrm{O}_{5}$ : C, 70.36; H, 4.97\%. Found: C, 70.20; H, 5.27\%.

7-Hydroxy-4-methoxy-10,10-dimethyl-10,11-dihydro-6H-naphtho[2,1-c]chromene-

6,12(9H)-dione (2o). Yield 55\%; mp 193-197 ${ }^{\circ} \mathrm{C}$; off-white solid; Selected IR frequencies (KBr): 3430 (broad, -OH), 3010 (C-H, aliphatic), 1665 (C=O, $\delta$-lactone), $1730 \quad(\mathrm{C}=\mathrm{O}$, naphthalenone), $1615\left(\mathrm{C}=\mathrm{C}\right.$, aromatic), 1045 (asymmetric and symmetric $\mathrm{C}-\mathrm{O}-\mathrm{C}$ stretching) $\mathrm{cm}^{-}$ ${ }^{1} ;{ }^{1} \mathrm{H} \mathrm{NMR}\left(\mathrm{CDCl}_{3}\right): \delta 1.11\left(6 \mathrm{H}, \mathrm{s}, 2 \times \mathrm{CH}_{3}\right), 2.70(2 \mathrm{H}, \mathrm{s}, \mathrm{C} 9-\mathrm{H}), 2.93\left(2 \mathrm{H}, \mathrm{s}, \mathrm{C}_{11}-\mathrm{H}\right), 3.40(3 \mathrm{H}$, $\left.\mathrm{s}, \mathrm{OCH}_{3}\right), 7.33-7.58(3 \mathrm{H}, \mathrm{m}, \mathrm{Ar}-\mathrm{H}), 8.19\left(1 \mathrm{H}, \mathrm{s}, \mathrm{C}_{8}-\mathrm{H}\right), 11.44\left(1 \mathrm{H}, \mathrm{s},-\mathrm{OH}\right.$ proton, $\mathrm{D}_{2} \mathrm{O}$ exchangeable); ${ }^{13} \mathrm{C}$ NMR: $\delta 28.5\left(2 \times \mathrm{CH}_{3}\right), 33.4(\mathrm{C}), 44.7\left(\mathrm{CH}_{2}\right), 54.8\left(\mathrm{CH}_{2}\right), 56.9\left(\mathrm{OCH}_{3}\right)$, $108.0(\mathrm{C}), 113.5(\mathrm{C}), 116.6(\mathrm{CH}), 121.1(\mathrm{CH}), 124.5(\mathrm{CH}), 127.7(\mathrm{C}), 128.3(\mathrm{C}), 136.7(\mathrm{CH})$, $143.4(\mathrm{C}), 151.5$ (C), 158.8 (C), $162.8 \quad\left(\mathrm{C}_{7}\right), 163.4 \quad(\mathrm{C}=\mathrm{O}, \delta$-lactone $), 200.6 \quad(\mathrm{C}=\mathrm{O}$, naphthalenone); Anal. Calcd. for $\mathrm{C}_{20} \mathrm{H}_{18} \mathrm{O}_{5}$ : C, 70.99; H, 5.36\%. Found: C, 70.66; H, 5.10\%.

\section{Acknowledgements}

The author expresses his sincere thanks to the Department of Advanced Organic Chemistry, P. D. Patel Institute of Applied Sciences, Charotar University of Science \& Technology (Charusat) for providing research facilities. Vaibhav Analytical Laboratory, Ahmedabad for IR spectral analysis as well as Oxygen Healthcare Research Pvt. Ltd., Ahmedabad for providing mass spectrometry facilities.

\section{References}

1. Soine, T. O. J. Pharm. Sci. 1964, 53, 231. Doi : 10.1002/jps.2600530302

2. Manolov, I.; Maichle-Moessmer, C.; Danchev, N. Eur. J. Med. Chem. 2006, 41, 882. Doi: 10.1016/j.ejmech.2006.03.007

3. Khan, I.; Kulkarni, M.; Sun, C. Eur. J. Med. Chem. 2005, 40, 1168. Doi: 10.1016/j.ejmech.2005.05.007

4. C. A. Kontogiorgis.; D. J. Hadjipavlou-Litina, J. Med. Chem. 2005, 48, 6400-6408. Doi: $10.1021 / \mathrm{jm} 0580149$ 
5. Reddy, N.; Reddy, M. M.; Cosenza, S.; Gumireddy, K.; Bell, C. S.; Reddy, P.; Reddy, M. R. Bioorg. Med. Chem. Lett. 2004, 14, 4093. Doi: 10.1016/j.bmcl.2004.05.016

6. Priepke, H.; Kauffimann-Hefinar, I.; Damm, K.; Schnapp, A. WO Patent 2003, 2003006443.

7. Vijaykumar, P. R.; Vinod R.; Rajeswar, R. V. Indian J. Chem. 2003, 42, 1738.

8. Venugopala, K. N.; Jayashree, B. S. Indian J. Heterocyclic Chem. 2003, 12, 307.

9. Vaccaro, W.; Yang, B.; Kim, S.; Huynh, T.; Leavitt K.; Li W. WO Patent 2004, 4009017.

10. Okumura, K.; Ashino, K.; Okuda, T. Yakugaku Zasshi 1962, 81, 1482, Chem. Abstr. 1962, $56,7938$.

11. Gingolani, G. M.; Gaultrieri, F.; Pigini J. Med. Chem. 1969, 12, 531.

12. Rao, B.; Mouli, C.; Reddy, Y. D. Ind. J. Chem. 1983, 2B, 176.

13. El-Naggar, A. M.; Ahmed, F. S.; Abd El-Salam, A. M.; Rady, M. A.; Latif, M. S. A. J. Heterocycl. Chem. 1981, 18, 1203. Doi: 10.1002/jhet.5570180627

14. Moustafa M. A. Scientica Pharmaceutica (Sci. Pharm.), 1991, 59, 213.

15. Kaczka, E. A.; Wolf, F. J.; Rathe, F. P; Folkers, K. J. J. Am. Chem. Soc. 1955, 77, 6404. Doi: 10.1021/ja01628a121

16. Smissman E. E.; Wilson C. O.; Gisvold, O.; Doerge, R. F. Textbook of organic Medicinal and Pharmaceutical Chemistry, 8th ed., P. 291-292, Lippincott Co.: Philadelphia, Toronto, 1982.

17. Sunil Kumar B., Thirupathi Reddy Y., Narsimha Reddy P., Kumar P. S., Rajitha B. J. Heterocyclic Chem., 2006, 43, 477. Doi: 10.1002/jhet.5570430233

18. El-Saghier A. M. M. and Al-Afaleq E. I.; Phosphorus, Sulfur and Silicon, 1998, 139, 67. Doi: 10.1080/10426509808035678

19. El-Saghier A. M. M. and Khodairy A.; Phosphorus, Sulfur and Silicon, 2000, 160, 105. Doi: 10.1080/10426500008043675

20. Patel, A. K.; Patel, N. H.; Patel, M. A.; Brahmbhatt, D. I. Arkivoc 2010, (xi), 28. 